\title{
Erleben und Unterstützungsbedarf von Eltern hospitalisierter Kinder
} mit Mehrfachbehinderung

\author{
Eine qualitative Studie
}

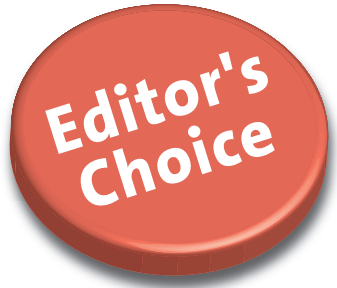

Brigitte Seliner ${ }^{1,2}$ (MNS, RN), Bea Latal' (Prof. Dr. med.), Rebecca Spirig ${ }^{1,3,4}$ (Prof., PhD, RN)

${ }^{1}$ Universitäts-Kinderspital Zürich, Schweiz

${ }^{2}$ Departement Pflegewissenschaft, Universität Witten/Herdecke, Deutschland

${ }^{3}$ Universitäts-Spital Zürich, Schweiz

${ }^{4}$ Institut für Pflegewissenschaft, Universität Basel, Schweiz

\begin{abstract}
Zusammenfassung:
Hintergrund: Die Hospitalisation eines Kindes mit Mehrfachbehinderung belastet Eltern, da sie auch im Spital viele Betreuungsaufgaben übernehmen müssen. Leider fehlt systematisches Wissen über das elterliche Erleben in dieser Situation und deren Unterstützungsbedarf.

Fragestellung: Wie erfahren Eltern die Hospitalisation, und welchen Unterstützungsbedarf geben sie für diesen Zeitraum an? Methode: An dieser qualitativen Untersuchung partizipierten 26 Elternteile (24 Mütter, zwei Väter) von 24 mehrfachbehinderten Kindern. Zwischen dem 1. Januar 2011 und dem 1. September 2013 wurden mit innen in einer universitären Kinderklinik halbstrukturierte Interviews geführt. Die strukturierende qualitative Inhaltsanalyse leitete die Analyse.

Ergebnisse: Für die Eltern stand die «Sorge um das Wohl des Kindes» im Zentrum. Die Einschätzung des kindlichen Wohls prägte das Ausmaß der «Arbeit der Eltern» und definierte den elterlichen «Unterstützungsbedarf» nach «Information und Schulung erhalten», «Bekannt sein und Kontinuität erfahren», «Ernst genommen werden und Mitsein erfahren», «Begleitet werden von erfahrenen Pflegenden» und «Entlastet und organisatorisch unterstützt werden».

Schlussfolgerung: Die Eltern leisten viel Arbeit, um das Wohl des hospitalisierten, mehrfachbehinderten Kindes sicherzustellen. Pflegefachpersonen können zur Entlastung dieser Eltern beitragen, indem sie deren Vertrauen in das Wohl des Kindes im Spital stärken. Dies gelingt, wenn Kontinuität und Kompetenz vonseiten der Pflegefachpersonen sichergestellt sind, wie es beispielsweise Pflegende mit einem Advanced-Practice-Hintergrund ermöglichen.
\end{abstract}

Schlüsselwörter: Kinder, Behinderung, Eltern, Krankenhaus, Erleben, Unterstützungsbedarf

\section{Experiences and support needs of parents of hospitalised children with multiple disabilities: A qualitative study}

\section{Abstract:}

Background: The hospitalisation of a multiple disabled child is stressful for parents because they continue to carry out demanding care procedures in hospital. Yet, systematic knowledge of the parental experience and of their support needs is missing. Question: How do parents experience the hospitalisation, and which support needs do they identify for this period?

Methods: Twenty-six parents (24 mothers, 2 fathers) of 24 children with multiple disabilities have participated in this qualitative study. Between 1 January 2011 and 1 September 2013, semi-structured interviews were conducted in a children's university hospital. A qualitative content analysis formed the basis for the analysis.

Results: "Concerns for the child's well-being" was central for the parents and focussed on the areas of "Pain", "Complications" and "Development". Perception of the child's well-being governs the extent of the "Parents' Work" and defines the parental "Support needs" to "Receive information and training", "Be known and experience continuity", "Be taken seriously and experience communion", "Be accompanied by experienced nurses", and "Be relieved and get organisational support".

Conclusion: Parents work hard to safeguard the well-being of their hospitalised child with multiple handicaps. Care professionals can ease the parents' burden by promoting parental confidence in their child's welfare in hospital. This will be successful if continuity of care and competence are ensured, for instance if it is provided by nurses with Advanced Practice background.

Keywords: children, disability, parents, hospital, experience, support needs 
Was ist (zu dieser Thematik) schon bekannt?

Die Hospitalisation eines behinderten Kindes belastet Eltern.

Was ist neu?

Pflegefachpersonen können die Eltern unterstützen, indem sie deren Vertrauen in das Wohl des Kindes im Spital stärken.

Welche Konsequenzen haben die Ergebnisse für die Praxis?

Verlangt werden Kontinuität und Kompetenzen, wie es Pflegefachpersonen mit einem Advanced-Practice-Hintergrund bieten.

\section{Hintergrund}

Kinder und Jugendliche mit Mehrfachbehinderungen leiden häufiger an Krankheiten, wie Atemwegsinfekten oder muskuloskelettalen Veränderungen, als gesunde Kinder und werden öfter, länger und mit erhöhtem Pflegeaufwand hospitalisiert (Hemsley, Kuek, Bastock, Scarinci \& Davidson, 2013). Zusätzlich hat die medizinische wie pflegerische Komplexität bei Kindern mit diesen Krankheitsbildern durch Fortschritte der Medizin und Technik zugenommen (Burns et al., 2010; Houtrow, Larson, Olson, Newacheck \& Halfon, 2014). Dies spiegeln Zahlen des Schweizerischen Bundesamtes für Statistik (2014) wider: Wurden 2011 beispielsweise knapp 4.000 Kinder mit Fehlbildungen und Chromosomenanomalien hospitalisiert, erhöhte sich diese Zahl im Jahr 2013 auf beinahe 6000 Kinder, mit einer Zunahme von mehr als 1000 Pflegetagen.

Die Pflege und Betreuung von Kindern, die entsprechend der ICF ${ }^{1}$ (WHO, 2010) durch Einschränkungen einer oder mehrerer Körperstrukturen bzw. -funktionen stark oder völlig in ihrer Selbstständigkeit limitiert und in der Teilhabe stark eingeschränkt sind, ist komplex und anspruchsvoll. In der Regel werden diese Leistungen im häuslichen Bereich von den Eltern erbracht (Ausserhofer, Mantovan, Pirhofer, Huber \& Them, 2009; Tadema \& Vlaskamp, 2010). Die Hospitalisation eines mehrfachbehinderten Kindes belastet die betroffenen Eltern zusätzlich, da sie viele Betreuungsaufgaben auch in der für sie fremden Umgebung übernehmen. Zusätzlich sorgen sie sich um ihr meist völlig abhängiges Kind, vor allem dann, wenn dessen verbale Kommunikation eingeschränkt ist. Die Eltern versuchen, es $\mathrm{zu}$ schützen und fühlen sich aufgrund ihrer Kompetenzen verantwortlich für die medizinisch-pflegerischen Aufgaben, was als belastender Dauerzustand erlebt wird und oft zu Erschöpfungszuständen führt (Avis \& Reardon, 2008; Iversen, Graue \& Clare, 2009; Iversen, Graue \& Raheim, 2013). Eltern fühlen sich häufig von medizinischen Fachpersonen nicht verstanden, vor allem wenn diese keine Erfahrung im Umgang mit behinderten Kindern mitbringen oder den Fokus mehr auf die technischen bzw. medizinischen Aspekte und weniger auf die kommunikative Ebene legen (Graham, Pemstein \& Curley, 2009; Robinson, Oxnam, Kelly, Broadbent \& Dillon, 1993). Insbesondere in komplexen Situationen sind die Kinder sowie die medizini- schen Fachpersonen auf die Unterstützung und Vermittlung der Eltern angewiesen (Ford \& Turner, 2001). Dabei darf nicht vergessen werden, dass vor allem die Mütter behinderter Kinder schon zu Hause erhöhter Belastung ausgesetzt sind und oft eine tiefe subjektive Lebensqualität angeben (Büker, 2010; Thyen, Sperner, Morfeld, Meyer \& Ravens-Sieberer, 2003). Letztere beschreibt die individuelle Wahrnehmung der spezifisch die Gesundheit betreffenden Lebensqualität und fokussiert auf die physischen, sozialen und psychischen Dimensionen des Wohlbefindens. Da die Eltern auf einzigartige Weise mit ihren Kindern verbunden sind, ist ihr eigenes Wohlergehen von zentraler Bedeutung für das Wohl des behinderten Kindes (Büker, 2010). Pflegefachpersonen (PFP) können dabei aufgrund ihrer Patientennähe eine wichtige Stütze für die Eltern sein (Bell, 2014; Olli, Vehkakoski \& Salantera, 2014). Leider fehlen Studien über Eltern hospitalisierter Kinder mit mehrfachen Behinderungen und deren Unterstützungsbedarf weitgehend. Für den deutschsprachigen Raum lässt sich aktuell keine Studie dazu finden (Seliner, Wattinger \& Spirig, 2015). Es ist daher unerlässlich, Problemfelder im Zusammenhang mit der Akutversorgung von Kindern mit schweren Behinderungen aus Sicht der Eltern zu identifizieren und den Unterstützungsbedarf zu erfassen, um gezielt pflegerische Hilfeleistungen zur Entlastung und Stärkung der Eltern anzubieten.

In der vorliegenden qualitativen Studie waren folgende Fragestellungen für uns leitend: Wie erleben Eltern die Hospitalisation ihres mehrfachbehinderten Kindes, und welchen Unterstützungsbedarf geben sie für diesen Zeitraum an?

\section{Methode}

\section{Design}

Die hier vorgestellte Untersuchung ist Teil einer MixedMethods-Studie zur Entwicklung und Evaluation pflegerischer Unterstützungsmaßnahmen für besagte Elterngruppe. Die Mixed-Methods-Studie mit sequentiellem Design umfasste als ersten Teil eine quantitative Untersuchung mittels Fragebogen, als zweiten eine qualitative Analyse mit individuellen Interviews und als dritten Teil eine Synthese (Creswell \& Plano Clark, 2011). Für die nachfolgend dargestellte qualitative Untersuchung führten wir halbstrukturierte Interviews durch, um die individuelle Sicht der Eltern und ihren Unterstützungsbedarf während der Hospitalisation ihres Kindes zu erforschen. Der qualitative Ansatz ermöglicht es hierbei, die Bedeutung individueller Erfahrungen in einem wenig erforschten Bereich mit einem methodisch kontrollierten Vorgehen zu erfassen und komplexe Zusammenhänge zu klären (Flick et al., 2005). 
Tabelle 1. Zentrale Themen aus dem Interviewleitfaden

1. Fragen zum elterlichen Erleben der Hospitalisation ihres Kindes

a) Wie erlebten die Eltern die Zeit mit ihrem Kind seit dem Eintritt?

b) Was waren besonders anstrengende Erlebnisse?

c) Was waren besonders erfreuliche Erlebnisse?

2. Rückfragen zur familiären Belastung und zur subjektiven Lebensqualität der Eltern (IFS, SF-36v2)

3. Rückfragen zur elterlichen Zufriedenheit mit der familienzentrierten Dienstleistung (MPOC-20)

4. Fragen nach dem Unterstützungsbedarf der Eltern während des Aufenthaltes

a) Welche Unterstützung war hilfreich?

b) Welche Form der Unterstützung hätte den Aufenthalt erleichtert?

5. Fragen zum Unterstützungsbedarf für das Kind aus Sicht der Eltern

a) Welche Form der Unterstützung haben die Eltern als hilfreich erlebt?

b) Welche Form der Unterstützung wünschen die Eltern für eine zukünftige Hospitalisation des Kindes?

\section{Setting, Untersuchungsgruppe und Datenerhebung}

Studienort war eine größere Universitäts-Kinderklinik der Deutschschweiz mit integrierter Neurorehabilitation für Kinder und Jugendliche. Die Datenerhebung erfolgte in den Bereichen der Intermediate Care (IMC), der Medizin, der Chirurgie und der Rehabilitation. Dort werden gemäß dem Spitalinformationssystem am häufigsten behinderte Kinder mit neurologischen Diagnosen betreut.

Für die Studie wurde der am häufigsten anwesende Elternteil von Kindern im Alter von einem bis 18 Jahren mit einer neurologischen Diagnose (z.B. Cerebral Parese), welche seit mindestens sechs Monaten vorlag, ausgewählt. Der Behinderungsgrad des Kindes musste gleich oder tiefer als $50 \%$ der altersentsprechenden Entwicklung gemäß der Functional Independence Measure für Kinder (UDSmr., 2009) sein. Erst wenn diese Kriterien erfüllt waren, wurden die Eltern durch die Studienkoordinatorin mündlich und schriftlich über die Studie informiert und angefragt. Die Eltern konnten entweder nur für den quantitativen oder zusätzlich auch für den qualitativen Teil der Gesamtstudie einwilligen. Für die Teilnahme an den Interviews mussten sich die Eltern auf Deutsch bzw. auf Englisch verständigen können oder mit dem Beisein einer Übersetzerin (für die Sprachen Italienisch, Portugiesisch, Griechisch, Türkisch, Serbokroatisch und Albanisch) einverstanden sein. Sobald die Zusage für ein Interview vorlag, kontaktierte die Studienleitung die Eltern.

Die halbstrukturierten Interviews führten wir anhand eines Leitfadens mit Fragen zum elterlichen Erleben der Hospitalisation ihres Kindes und zum Unterstützungsbedarf durch (siehe Tabelle 1). Des Weiteren wurden die im Rahmen des quantitativen Studienteils abgegebenen Fragebogen besprochen, um die jeweiligen Themen zu vertiefen und die Hintergründe für die Antworten auf die standardisierten Instrumente zu eruieren. Bei den Fragebogen handelte es sich um den SF-36v2 (Ware, 2000), den IFS (Ravens-Sieberer et al., 2001) und den MPOC-2O (Cunningham \& Rosenbaum, 2014), die in Tabelle 2 näher erklärt werden. Ferner wurden demografische und medizinische Daten der Kinder und der Eltern erfasst.

Die Interviews dauerten durchschnittlich 38 Minuten (SD: 9.5). Die Gespräche wurden größtenteils in Mundart geführt und auf Tonträger aufgenommen. Anschließend wurden sie in Standarddeutsch transkribiert und in das Datenmanagementsystem ATLAS.ti 7.2@ exportiert.

Bis auf zwei Interviews, die später stattfanden, wurden alle Befragungen im Zeitraum der Hospitalisation zwischen dem 1. Januar 2011 und dem 1. September 2013 geführt.

\section{Datenanalyse}

Die 24 Interviews werteten wir mittels der strukturierenden qualitativen Inhaltsanalyse aus (Mayring, 2007). Diese ermöglicht es, den Inhalt von Kommunikationsmaterial systematisch in spezifischen Schritten zu reduzieren, zu abstrahieren, zu explorieren und zu strukturieren. Das primäre Kodiersystem wurde deduktiv, basierend auf dem Interviewleitfaden, erarbeitet. Die weitere Analyse erfolg-

Tabelle 2. Beschreibung der standardisierten Fragebogen

\begin{tabular}{|c|c|c|c|}
\hline Fragebogen & Thema & Subkonzepte & Autoren \\
\hline $\begin{array}{l}\text { SF-36v2 } \\
\text { (Short Form 36, Version 2) }\end{array}$ & $\begin{array}{l}\text { Gesundheitsbezogene Lebens- } \\
\text { qualität }\end{array}$ & $\begin{array}{l}36 \text { Fragen zu den } 2 \text { Hauptbereichen psychi- } \\
\text { sche und körperliche Gesundheit }\end{array}$ & Ware, 2000 \\
\hline $\begin{array}{l}\text { IFS } \\
\text { (Impact of Family Scale) }\end{array}$ & Familiäre Belastung & $\begin{array}{l}33 \text { Fragen zu den } 5 \text { Subkonzepten (tägl. soziale } \\
\text { Belastung, persönliche Belastung, finanzielle } \\
\text { Belastung, Belastung der Geschwisterkinder, } \\
\text { Probleme bei der Bewältigung) }\end{array}$ & Ravens-Sieberer et al., 2001 \\
\hline $\begin{array}{l}\text { MPOC- } 20 \\
\text { (Measure of Process of Care) }\end{array}$ & $\begin{array}{l}\text { Zufriedenheit mit der familien- } \\
\text { zentrierten Dienstleistung }\end{array}$ & $\begin{array}{l}20 \text { Fragen zu den } 5 \text { Subkonzepten (Befähi- } \\
\text { gende und partnerschaftliche Betreuung, } \\
\text { Anbieten von generellen und spezifischen } \\
\text { Informationen, koordinierte und umfassende } \\
\text { Betreuung sowie respektvolle und unterstüt- } \\
\text { zende Betreuung) }\end{array}$ & Cunningham \& Rosenbaum, 2014 \\
\hline
\end{tabular}


Tabelle 3. Demografische Daten der Eltern

\begin{tabular}{lc}
\hline Eltern & $\mathrm{N}=26$ \\
\hline Alter (Jahre), $M \pm S D$ & $40,7 \pm 7,6$ \\
Elternteil, N (\%) & \\
$\quad$ Mutter & $24(92,3)$ \\
$\quad$ Vater & $2(7,7)$ \\
Anzahl Geschwisterkinder, M (Range) & $2(0-4)$ \\
Zivilstand, N (\%) & \\
$\quad$ Partnerschaft/verheiratet & $24(92,3)$ \\
$\quad$ Single & $2(7,7)$ \\
Migrationshintergrund, N (\%) & \\
$\quad$ Schweizer & $17(65,4)$ \\
Andere & $9(34,6)$ \\
Sprache, $N$ (\%) & \\
$\quad$ Deutsch & $25(96,2)$ \\
Englisch & $1(3,8)$ \\
\hline
\end{tabular}

te induktiv anhand der zentralen Interviewinhalte. Identifizierte Textstellen wurden Satz für Satz auf das Wesentliche reduziert, auf eine sprachliche Ebene gebracht und abstrahiert. Mittels Visualisierung und des Schreibens von Memos konnten erkennbare Muster ausgemacht und einem Kategoriensystem zugeordnet werden. Die Analyse führte die Erstautorin zusammen mit vier Forschungspraktikantinnen durch und validierte die Daten diskursiv innerhalb der Forschungsgruppe und mit der Letztautorin.

\section{Ethik}

Die Ethikkommission des zuständigen Kantons (KEK) bewilligte die Gesamtstudie, und alle Teilnehmenden unterschrieben die Einwilligungserklärung.

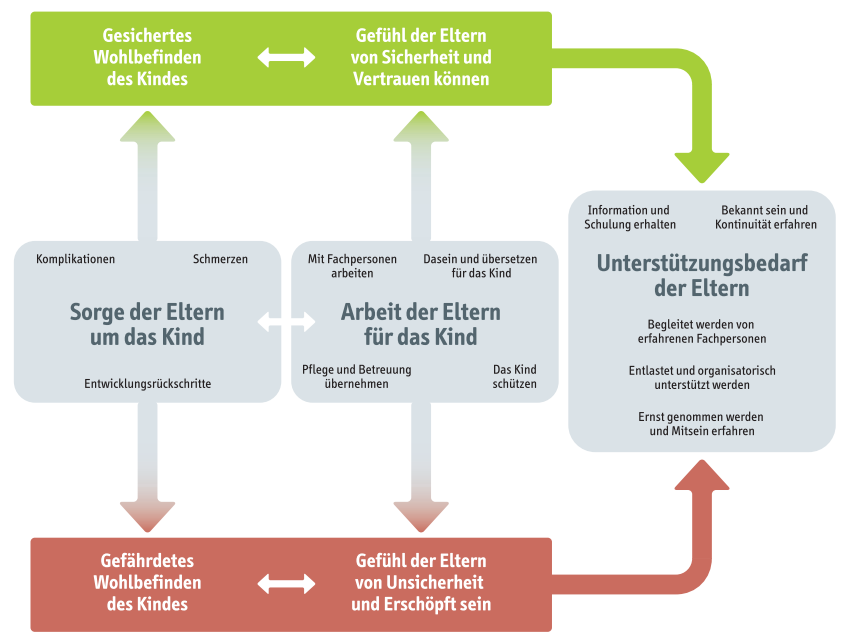

Abbildung 1. Erleben und Unterstützungsbedarf der Eltern.
Tabelle 4. Demografische und medizinische Daten der Kinder

\begin{tabular}{|c|c|}
\hline Kinder & $N=24$ \\
\hline Alter (Jahre), $M \pm S D$ & $8,7 \pm 5,3$ \\
\hline \multicolumn{2}{|l|}{ Geschlecht, N (\%) } \\
\hline männlich & $15(62,5)$ \\
\hline weiblich & $9(37,5)$ \\
\hline Anzahl Diagnosen, $M \pm S D$ & $6,7 \pm 2,6$ \\
\hline Grad der Behinderung $\left(\right.$ WeeFIM $\left.{ }^{\circledast}\right), M \pm S D$ & $27,5 \pm 11,5$ \\
\hline \multicolumn{2}{|l|}{ Neurologische Diagnose, N (\%) } \\
\hline Zerebralparese & $12(50,0)$ \\
\hline Genetisches Syndrom & $4(16,7)$ \\
\hline Andere (z.B.Trisomie, Epilepsie) & $4(16,7)$ \\
\hline Muskeldystrophie & $3(12,5)$ \\
\hline Traumatische Hirnverletzung & $1(4,2)$ \\
\hline \multicolumn{2}{|l|}{ Hospitalisationsgrund, N (\%) } \\
\hline Orthopädische Eingriffe & $12(50,0)$ \\
\hline Chirurgische Eingriffe (z.B. Urologie) & $4(16,7)$ \\
\hline Untersuchungen/Abklärungen & $3(12,5)$ \\
\hline Rehabilitation & $3(12,5)$ \\
\hline AZ Verschlechterung (z.B. Infektion) & $2(8,3)$ \\
\hline
\end{tabular}

\section{Ergebnisse}

26 Elternteile, respektive 24 Mütter und zwei Väter, von insgesamt 24 hospitalisierten Kindern (15 Buben, neun Mädchen) mit Mehrfachbehinderungen partizipierten an der Teilstudie. Von zwei Kindern nahmen sowohl Mutter als auch Vater teil. Die meisten Teilnehmenden waren verheiratet (92,3\%). Zwei Mütter (7,7\%) waren alleinerziehend. Etwa ein Drittel brachte einen Migrationshintergrund mit, und nur eine Mutter (3,8\%) wünschte, das Interview auf Englisch zu führen. Die Kinder wiesen durchschnittlich 6,7 Diagnosen mit einer primär neurologischen Erkrankung auf. Die demografischen Daten der Eltern werden in Tabelle 3 und diejenigen der Kinder in Tabelle 4 aufgeführt.

Die Analyse ergab drei zentrale, sich gegenseitig beeinflussende Hauptkategorien mit den jeweiligen Subkategorien, die das elterliche Erleben der Hospitalisation, ihre Arbeit und ihren Unterstützungsbedarf darstellen (Abbildung 1).

Die erste Kategorie beschreibt die in den Interviews geäußerte «Sorge der Eltern um das Wohl des Kindes» hinsichtlich der Themen «Schmerzen», «Komplikationen» und «Entwicklungsrückschritte». Die Sorge um das kindliche Wohl, das die Eltern zwischen «gefährdet» und «gesichert» beschrieben, zeigte Auswirkungen auf die von ihnen erbrachten Leistungen, beschrieben in der zweiten Kategorie «Arbeit der Eltern für das Kind». Dieser sind die Subkategorien «Dasein und übersetzen für das Kind», «Pflege und Betreuung übernehmen», «Das Kind schützen» und «Mit Fachpersonen arbeiten» zugeordnet. Das Ausmaß der beschriebenen Arbeit hing stark von der elterlichen Wahrnehmung des Kindeswohls ab. Je verlässlicher Letzteres gesichert war, desto mehr beschrieben die Eltern 
ein «Gefühl von Sicherheit und Verantwortung abgeben können» bzw. nahm im umgekehrten Fall das «Gefühl von Unsicherheit und sich erschöpft fühlen» zu. Diese von den Eltern beschriebenen Ausprägungen der Sorge und das damit verbundene Ausmaß an Eigenleistung, die in Kategorie eins und zwei zusammengefasst sind, verweisen auf die dritte Kategorie, den von den Eltern geäußerten «Unterstützungsbedarf». Dieser Bedarf wird anhand der Subkategorien «Information und Schulung erhalten», «Bekannt sein und Kontinuität erfahren», «Ernst genommen werden und Mitsein erfahren», «Begleitet werden von erfahrenen $\mathrm{PFP}$ » und «Entlastet und organisatorisch unterstützt werden» beschrieben.

\section{Sorgen der Eltern um das Wohl des Kindes}

Die Eltern sorgten sich sehr um das Wohl und die Sicherheit ihres mehrfachbehinderten Kindes im Spital und fühlten sich dafür verantwortlich. Sie nahmen ihr Kind als verletzlich wahr, vor allem aufgrund der häufig eingeschränkten Kommunikation, aufgrund dessen Hilflosigkeit sowie der fragilen Gesundheit. Sie mussten sich zuerst ein Gefühl von Sicherheit und Vertrauen erarbeiten, bevor sie sich zurücknahmen und Verantwortung abgeben konnten (P14; Ab. 59): «Wenn ich nicht sicher bin, dass er gut aufgehoben ist, kann ich nicht ruhig schlafen. Ich sehe alles, auch wenn es nie passieren wird.» Die Eltern litten mit, wenn sich der Zustand des Kindes verschlechterte (P15; Ab. 11): «Wenn Sie mich fragen, wie es mir geht, sage ich immer, wenn es $S$. gut geht, dann geht es mir auch gut. Ich leide einen Teil mit ihr mit.» Nicht überraschend vermittelte die kontinuierliche Präsenz der Fachpersonen der IMC Sicherheit (P4; Ab. 28): «Aufder IMC kann ich mich eher lösen, kann gehen, weil ich weiß, die Pflege ist da.» Gleichwohl erfuhren viele Eltern erst durch die Verlegung des Kindes in die Rehabilitation eine Entlastung. Zwar benötigten sie bei Erstaufenthalten oder nach anstrengenden Akutaufenthalten Zeit, um Vertrauen aufzubauen; in der Folge konnten sie aber Verantwortung abgeben (P7; Ab. 9): «Zu wissen, mein Kind ist gut aufgehoben, es wird ihm gut geschaut, er kommt womöglich gar in einem besseren Zustand nach Hause, das ist sehr beruhigend. Ich kann auch mal die Verantwortung abgeben.» Natürlich gab es auch positiv erlebte Behandlungen wie beispielsweise die Entfernung der Trachealkanüle, welche die Selbstständigkeit und das Wohlbefinden des Kindes förderte. Dennoch, die Eltern sorgten sich stets um die gesundheitliche Stabilität des Kindes und versuchten vor allem, Schmerzen, Komplikationen und Entwicklungsrückschritte zu verhindern.

\section{Schmerzen}

Potenzielle Schmerzzustände ihres Kindes erlebten die Eltern bereits im Vorfeld des Spitalaufenthaltes als sehr belastend. Ihnen war bewusst, dass das Kind Schmerzen nicht unmittelbar ausdrücken konnte, bzw. dass sein Verhalten schwierig zu interpretieren war. Eine Mutter äußer- te sich folgendermaßen (P4; Ab. 218): «Schmerz kann man mit ihr nicht vorbereiten. Und es gibt in der Situation keine Floskeln wie 〈es wird schon gut> und <es ist nicht so schlimm>. Das nützt nicht mal bei normalen Kindern. Dies war in meiner persönlichen Auseinandersetzung das Schwierigste.» Einige Eltern hatten bezüglich Schmerzeinschätzung eine gewisse Hilflosigkeit bei den Fachpersonen beobachtet (P4; Ab. 70): «Die Ärzte haben sie permanent gefragt, hast du Schmerzen, und sie schreit NEIN. Okay, sie hat keine Schmerzen. Da musste ich sagen, doch sie hat Schmerzen, sonst würde sie nicht schlagen [...].» Gerade weil die meisten Kinder ihre Schmerzen nicht verbal mitteilen konnten und ihre Reaktionen schwierig zu interpretieren waren, zeigten sich Eltern diesbezüglich besorgt und wachsam gegenüber Schmerztherapien.

\section{Komplikationen}

Viele Eltern befürchteten aufgrund der fragilen Gesundheit ihres Kindes Komplikationen oder hatten solche bereits erlebt. Vor allem das geschwächte Immunsystem und das damit einhergehende Infektionsrisiko beunruhigte sie. Komplikationen bedeuteten meist, dass sich der allgemeine Gesundheitszustand des bereits beeinträchtigten Kindes verschlechterte und sich die Erholung verzögerte. Komplikationen konnten die ganze Familie belasten, wie eine Mutter erzählte (P11; Ab. 212): «Es dauert sehr lange, bis er wieder zu sich kommt [...]. Wenn er krank ist, kann er nicht schlucken, nicht essen, und er wird sehr schwach. Dann schläft er nicht. Nichts funktioniert mehr. Und wir sind alle, nicht nur L., die ganze Familie wird krank. Ich vor Müdigkeit, und L. ist richtig krank.»

\section{Entwicklungsrückschritte}

Den Eltern war die Entwicklung ihres Kindes wichtig. Dessen Stärken sollten erkannt und gefördert werden. Die Eltern schilderten, dass das Kind sich zuweilen langweilte und die ungewohnte Klinikumgebung es stärker einschränkte als gewohnt. Eine Sorge war, dass das Kind nicht aktiv in den Alltag einbezogen wurde (P14; Ab. 135): «Ich weiß, dass wir ihn nicht zum Gehen bringen. [...] Aber ich denke, niemand soll nur sitzen und warten auf irgendwas.» Mit Sorge wurden während oder nach der Hospitalisation Rückschritte in der Entwicklung oder Verhaltensänderungen beobachtet (P5; Ab. 110): «Sie ist auch viel stiller geworden. Das ist sehr schwierig, weil sie nicht viel oder nur ein paar Worte spricht.»

\section{Arbeit der Eltern für das Wohl des Kindes}

Je mehr sich die Eltern sorgten und sich verantwortlich fühlten, desto größer war die erbrachte Leistung, um das kindliche Wohlbefinden sicherzustellen - oder umgekehrt, desto mehr konnten sie sich zurücknehmen und Verant- 
wortung abgeben. Dies begann mit der Spitalvorbereitung und endete meist erst mit dem Austritt.

\section{Dasein und übersetzen für das Kind}

Viele der befragten Eltern waren während des gesamten Spitalaufenthaltes anwesend, sicher tagsüber, teils 24 Stunden. Sie erachteten ihre Präsenz beim Kind als notwendig und versuchten, dem Kind das Gewohnte zu vermitteln bzw. das Ungewohnte zu übersetzen, um damit den Genesungsprozess bestmöglich zu unterstützen. Gerade erfahrene Eltern wussten um die medizinische Komplexität des Krankheitsbildes ihres Kindes und wie schwierig und aufwendig es für Fachpersonen ist, die Gesamtsituation $\mathrm{zu}$ erfassen (P5; Ab. 90): «Sprechen tut sie nicht so viel oder nur ein paar Worte. Das heißt, man muss sich sehr einlassen. Das braucht sehr viel Zeit. [...] Und ich denke, das ist anspruchsvoll für das Pflegepersonal», oder wie eine Mutter sagte (P9; Ab. 45): «Ich bin 24 Stunden mit L. zusammen, und ich kenne ihn wirklich am besten. Wenn eine Fachperson ihn nicht kennt und nur Berichte gelesen hat, kann sie nicht wissen, wieso und warum.»

\section{Pflege und Betreuung übernehmen}

Die Mütter übernahmen die Pflege und Betreuung ihres hospitalisierten Kindes mit großer Selbstverständlichkeit. Sie brachten dafür viel Erfahrung und Wissen mit und nahmen wahr, dass vor allem die Pflegenden der Akutabteilungen sehr viel zu tun hatten. Eine Mutter berichtete (P10; Ab. 504): «Eine Pflegende hat gesagt, aber Sie machen ja alles. Da habe ich gesagt: $<$ Soll ich daneben stehen und zusehen, wie Sie das Kind katheterisieren? [...] Nicht weil ich denke, Sie können es nicht, sondern weil ich sehe, Sie haben andereszutun〉.» Eine Mutter erhoffte sich, mit ihren Leistungen die Unterstützung der PFP auch während ihrer Abwesenheit zu sichern (P16; Ab. 99): «Mein Ziel ist, dass ich, wenn ich da bin, so viel wie möglich übernehme. Sodass, wenn ich stundenweise zum Arbeiten gehe, die Pflege das übernimmt. So quasi als Gegenzug.»

\section{Das Kind schützen}

Denjenigen Eltern, die Fehler oder Komplikationen erlebt hatten, war Kontrolle wichtig. Dabei schienen Unsicherheiten, gerade in neuen Situationen, zum Erleben der Eltern dazuzugehören, wie eine Mutter berichtete (P16; Ab. 139): «Wenn man neu auf einer Station ist, gehen einfach gewisse Dinge vergessen. Wir sagen zum Beispiel, man muss die Milch eindicken. Dann kommen sie trotzdem mit Milch, die nicht eingedickt ist.» Einige Eltern entwickelten ein systematisches Vorgehen, um die Sicherheit des Kindes zu gewährleisten, wie eine Mutter erzählte (P2; Ab. 274): «Und aus der Situation [Medikamentenfehler] ist es für mich Standard geworden, bei jedem Eintritt, sobald alle Informationen übertragen sind, um fünf Minuten zu bitten, damit wir zusammen die Dokumentation vergleichen können.» Auch ohne diesbezügliche Erfahrungen bringen die Eltern ein gewisses Maß an Misstrauen mit und geben ihr Bestes, um das Kind zu schützen (P18; Ab. 70): «Wenn ich selbst das Bestmögliche mache, dann kann ich mir danach keinen Vorwurf machen. Dann habe ich das Maximum gegeben.»

\section{Mit Fachpersonen arbeiten}

Mit den Fachpersonen zusammenzuarbeiten, sie zu unterstützen und zwischen den Bereichen zu vermitteln, war für die Eltern ein zentrales Anliegen, um eine optimale Pflege und Behandlung für ihr Kind zu gewährleisten. So berichtete eine Mutter (P2; Ab. 247): «Unterstützen ist ein Schlüsselfaktor [...]. Ich schreibe die Dokumentation mit wichtigen Tipps oder Informationen, damit die Pflegenden sich unterstützt fühlen. Wir stehen auch jederzeit zur Verfügung.» Ein Teil der Zusammenarbeit bestand darin, Informationen weiterzuleiten und zwischen den Fachpersonen zu vermitteln. Vor allem die fehlende Kontinuität erforderte gemäß den Eltern viel Aufmerksamkeit (P22; Ab. 87): «Ich muss den Leuten erklären, wie mein Kind funktioniert. Es kann nicht sprechen. Bei den vielen Wechseln muss man es immer wieder erklären, weil der Wissensstand nicht gut weitergegeben wird.»

\section{Unterstützungsbedarf der Eltern}

Der Unterstützungsbedarf der Eltern zielte direkt oder indirekt auf das Wohlergehen des Kindes und sollte ihre Arbeit erleichtern und ihre eigene Kompetenz für die Pflege im Spital wie zu Hause fördern. Je nach gesundheitlicher Situation des Kindes und dem elterlichen Gefühl von Sicherheit ist mehr oder weniger Unterstützung nötig.

\section{Information und Schulung erhalten}

Frühzeitige, direkte und nachvollziehbare Information war den Eltern wichtig, um die bevorstehenden Schritte für sich und das Kind einzuschätzen und die Kontrolle zu wahren. Der Informationsbedarf war entsprechend der individuellen Situation des Kindes und dem Erfahrungshintergrund der Eltern vielfältig. Grundsätzlich wünschten sie, über Veränderungen des Therapiemanagements, den Heilungsverlauf und den Austritt, aber auch über die Unterbringung sowie die Finanzierung informiert zu werden. Im besten Falle beginnen Information und Austausch vor dem Eintritt mit einer kompetenten Fachperson, wie eine Mutter darlegte (P22; Ab. 287): «Es wäre super, wenn man eine Kompetenzperson hätte für Kinder mit Behinderungen. Die dann alles im Voraus einholen würde. [...] So hätte man Zeit, sich vorzubereiten.» Die Eltern schätzten ehrliche Informationen. Diese würden gemäß einer Mutter helfen, sich auf Situationen einzustellen (P19; Ab. 25): «Wenn mir gesagt worden wäre, hören Sie, es geht nicht anders. Sie werden als Eltern gebraucht [...]. Dann wäre dies so.» Vor allem in 
Situationen, in denen große interprofessionelle Teams involviert waren, wünschten sich die Eltern einen funktionierenden Informations- und Kommunikationsfluss. War dieser gewährleistet, fühlten sie sich sicher (P9; Ab. 107): «Ich fühle mich sicher, wenn die verschiedenen involvierten Ärzte und Therapeuten gut zusammenarbeiten und gut miteinander kommunizieren.»

Sobald sich der Austritt näherte, schätzten die Eltern frühzeitige Informationen und vor allem eine Schulung durch die Therapeuten und die PFP. Diese sollten möglichst auf die konkrete Situation zu Hause ausgerichtet sein, wie z. B. ein angepasster Transfer.

\section{Bekannt sein und Kontinuität erfahren}

Den Eltern gab das Bekanntsein auf einer Station Sicherheit. Entsprechend verringerte sich der Aufwand sowohl für sie als auch für die Fachpersonen. Eine Mutter berichtet, wie sie dank Kontinuität durch die Pflegefachpersonen Vertrauen entwickelte und Entlastung erfuhr (P2; Ab. 27, 50): «Es gibt mir großes Vertrauen, dass sie bei der Planung schauen, dass diejenigen sie betreuen, die L. kennen. Sie wissen genau, wofür die Jejunalsonde ist und wie das Verstopfen zu vermeiden ist [...]. Ich muss nichts sagen, nicht daran erinnern. Ich spüre, dass sie sich vertraut fühlen im Umgang mit L.»Das Bekanntsein führte gemäß den Eltern zu einer pflegerischen Qualitätssteigerung (P16; Ab: 189): «Man merkt, sie versuchen zu überlegen, wo unsere Tochter am besten hinkommt. Ich habe das Gefühl, sie wissen, wer wir sind [...]. Dieses Mal hatten sie schon einiges vorbereitet.» Kontinuität durch die Pflegefachpersonen erlaubte es den Eltern auch, sich zurückzunehmen (P4; Ab. 64): «Ideal wäre, wenn die Pflegefachperson wüsste, da kommt ein mehrfachbehindertes Kind mit auch sprachlichen Schwierigkeiten. Dann müsste es fast eine 1:1-Betreuung geben. Die Eltern wüssten, diese lernt mein Kind kennen, und ich kann mich zurückziehen.»

\section{Ernst genommen werden und Mitsein erfahren}

Viele Eltern fühlten sich erleichtert, wenn ihre Expertise, ihre Beobachtungen und Einschätzungen ernst genommen wurden. Ihnen wurde gesagt, dass ihr Wissen unerlässlich sei. Entsprechend wünschten sie eine Mitsprachemöglichkeit (P18; Ab. 7): «Ich möchte, dass die Ärzte auf uns eingehen, wenn wir sehen, etwas ist nicht gut. [...] Es ist uns häufig gesagt worden, dass wir unsere Tochter am besten kennen und wissen, was gut für sie ist und was nicht. Dann ertrage ich es nicht, wenn man einfach nach Buch geht, weil es ein Spital ist oder weil Leute lernen müssen.» Die Teilnahme an der Visite wurde als ein Weg genannt, um vermehrt bei Entscheidungsprozessen miteinbezogen zu werden. Ernst genommen zu werden bedeutete für die Eltern auch, in schwierigen emotionalen Situationen nicht alleine gelassen zu werden und Mitsein und Zuwendung zu erfahren (P4; Ab. 22): «Auch wenn man nicht helfen kann, denke ich, bleibt doch bitte einfach da und hört mir zu, oder wir tauschen uns aus.» Viele Eltern waren tagelang mit ihrem Kind im Spital und traten schon erschöpft mit dem Kind in die Klinik oder in die Rehabilitation ein. Ein Umstand, den sie von den Fachpersonen anerkannt haben möchten und der eine feinfühlige Kommunikation erfordert. Diese muss nicht ausschließlich von PFP geleistet werden, jedoch tragen diese maßgeblich dazu bei, dass sich die Eltern auf den Stationen aufgehoben und verstanden fühlen.

\section{Begleitet werden von erfahrenen Pflegefachpersonen}

Die Eltern wünschten sich spezifisches Wissen und Erfahrung - primär von den Pflegefachpersonen - im Umgang mit dem behinderten Kind. Dies betraf die Fähigkeit, mit dem Kind Kontakt aufzunehmen und zu kommunizieren, mit seinem Verhalten umzugehen und es zu fördern. Des Weiteren sollten die Betreuenden über die Fähigkeit verfügen, die individuelle Situation des Kindes als Ganzes zu erfassen und Veränderungen frühzeitig zu erkennen. Die Eltern schätzten erfahrene Fachpersonen, die in komplexen Situationen die Initiative ergriffen (P2; Ab. 47): «Schlussendlich, in der Nacht, da war ein Engel da, denn die Nachtwache ist zurück zu der Umrechnung vom Morphin gegangen. Bei der Umrechnung ist ein schwerer Fehler passiert. L. hat einen Zehntel von ihrer normalen Dosis bekommen.» Das Eingestehen von Grenzen vonseiten der Fachpersonen empfanden die Eltern nicht als Schwäche. Es gab ihnen die Sicherheit und das Vertrauen, dass sich diese bei Fragen melden würden und das Kind ernst nähmen, wie eine Mutter darstellte (P10; Ab. 436): «Die haben gesagt: 〈Wenn wir in der Nacht nicht weiter wissen, rufen wir Sie an.〉 [...] Und das hat mir ein gutes Gefühl gegeben. Ich habe gewusst, sie nehmen das Kind ernst, und wenn sie nicht weiterkommen, rufen sie an.»

\section{Entlastet und organisatorisch unterstützt werden}

Trotz der vielen Leistungen, die Eltern selbstverständlich erbrachten, waren sie auf Entlastung in der Pflege und der Betreuung ihres Kindes im Spital angewiesen. Je nach $\mathrm{Zu}$ stand des Kindes sowie körperlicher und mentaler Fitness der Eltern wünschten sich diese mehr oder weniger Unterstützung (P19; Ab. 21, 25): «Ich hatte gehofft, dass man zwei bis drei Stunden Pause haben darf. Es wäre mir egal, wenn ich kein behindertes Kind hätte und nicht die ganze Zeit akut am Limit wäre. Aber da ich schon zu Hause Unterstützung brauche, damit ich es schaffe ...» Für viele Eltern war es nicht so sehr das konkrete Übernehmen von Handlungen, das entlastete, sondern das aktive Anbieten oder Planen von Pausen oder das gemeinsame Pflegen. Gezieltes Fördern des Kindes durch regelmäßige Besuche der Heilpädagogin oder wenn sich jemand vom Team Zeit zum Spielen nahm, bzw. Möglichkeiten dazu organisierte, entlastete zusätz- 
lich. Vor allem Eltern von sehr unruhigen Kindern wünschten sich eine Organisation, die lange Notfallaufenthalte verhinderte und Aufnahmen direkt auf «ihrer» Station erlaubte. Einzelzimmer schätzten die Eltern zum Schutz der Kinder vor Infektionen, Unruhe und neugierigen Blicken anderer Eltern.

\section{Diskussion}

Die hier vorgestellte qualitative Untersuchung zeigt erstmals für den deutschsprachigen Raum auf, wie pflegende Eltern - vor allem die Mütter - die Hospitalisation ihres Kindes mit Mehrfachbehinderung erleben und welchen Unterstützungsbedarf sie haben.

Die Ergebnisse verdeutlichen, dass für die Eltern die Sorge um das Wohl des Kindes zentral ist. Obwohl sich die Eltern von der Hospitalisation eine Verbesserung des Gesundheitszustandes ihres Kindes erhofften, fürchteten sie damit einhergehende Schmerzen, Komplikationen oder Entwicklungsrückschritte. Dieser Zwiespalt wird auch in anderen qualitativen Studien beschrieben und als Ursache für die emotionale Anspannung der Eltern während eines Spitalaufenthaltes angegeben (Iversen et al., 2013). Gemäß diesen Autoren fühlen sich die Eltern verantwortlich für die Hospitalisation und das damit allfällige Leiden, das dem völlig von ihnen abhängigen Kind widerfahren mag. Oulton und Heyman (2009) begründen dies damit, dass die Eltern im häuslichen Bereich die Verantwortung für die hochspezialisierte Pflege und Betreuung ihrer Kinder tragen und dadurch eine «ängstliche Verantwortung» beibehalten, auch wenn sie die Pflege und Betreuung zeitweise anderen Fachgruppen überlassen.

Aufgrund dieser Faktenlage erstaunt es nicht, dass sämtliches Handeln der befragten Eltern auf das Wohlergehen und die Sicherheit des Kindes ausgerichtet war. Es beeinflusste ihr eigenes Sicherheitsgefühl und die Bereitschaft, Verantwortung abgeben zu können oder, im ungünstigen Fall, sich zunehmend erschöpft zu fühlen. Dabei fällt auf, dass diese Eltern, konkret die Mütter, auch wenn sie sich erschöpft und müde fühlten, weiterhin die Pflege und Betreuungsaufgaben übernahmen. Im Gegensatz zu Eltern gesunder Kinder ist für diese Eltern die Erholung zu Hause jedoch erschwert, was die bereits einleitend erwähnte tiefe subjektive Lebensqualität der Mütter behinderter Kinder verstärken mag (Phua, Reid, Walstab \& Reddihough, 2005).

Da diese Kinder häufig wiederholt hospitalisiert werden und die Literatur von einer hohen Belastung der Eltern ausgeht, war es uns wichtig, deren Unterstützungsbedarf zu erfassen. Die Ergebnisse zeigen, dass das Hilfsangebot sowohl auf das Kind wie auch auf die Eltern ausgerichtet sein sollte, und der Bedarf wiederum direkt oder indirekt auf das Wohlbefinden des Kindes zielt. Es war den Eltern wichtig, dass das Kind Geborgenheit erfährt und ihm «gut geschaut» wird, im Sinne der Fürsorge und dem Sich einlassen von PFP, wie es Benner und Wrubel schon 1997 an- hand des Caringmodells beschrieben. Viele Eltern wünschten sich gerade in anhaltend schwierigen Situationen diese Fürsorge und das emphatische Mitsein von PFP.

Eine zentrale Rolle nimmt die Kontinuität der PFP sowie ihre Kompetenz im Umgang mit dem behinderten Kind ein. Wie bei Oulton und Heyman (2009) beschrieben, übertrugen die Eltern in der vorliegenden Studie einer Fachperson die Verantwortung nur, wenn diese sich außerordentlich kompetent in der Pflege des Kindes gezeigt hatte. Die Eltern mussten sich sicher sein, dass sich die Fachperson genügend idiografisches Wissen angeeignet hatte, um die individuelle und komplexe Pflege durchführen zu können. Fehlte das Vertrauen, nahmen die Eltern hohe Belastungen für das Kind in Kauf.

Um Vertrauen in die Kompetenzen der Fachpersonen entwickeln zu können, waren den befragten Eltern ein guter Informationsfluss, empathische Kommunikation und konkrete Schulung wichtig. Dieser Bedarf ist aus anderen Studien von Eltern behinderter Kinder bekannt (Seliner, Wattinger \& Spirig, 2015). Es lässt sich daher fragen, ob dies nicht auch Ausdruck einer immer noch mangelhaften partnerschaftlichen Zusammenarbeit zwischen Fachpersonen und diesen Eltern ist. In dieselbe Richtung weist das elterliche Bedürfnis, ernst genommen zu werden und Zuwendung zu erfahren - allesamt Kernelemente der partnerschaftlichen Zusammenarbeit, wie sie in der familienzentrierten Pflege und Betreuung beschrieben werden (Mikkelsen \& Frederiksen, 2011). Ebenso bemängeln die Eltern eine Mitsprachemöglichkeit bei Entscheidungen über Behandlung und Pflege. Die fehlende Mitsprache steht im Widerspruch zur wahrgenommenen Verantwortungslast der Eltern. Dies mag damit zusammenhängen, dass die Rolle der Laienpflege durch Angehörige und Eltern in der Betreuung behinderter Menschen nach wie vor nicht geklärt ist (Ausserhofer et al., 2009; De Geeter, Poppes \& Vlaskamp, 2002).

\section{Limitationen}

Die vorliegende Studie wurde nur an einem universitären Kinderspital durchgeführt, und gewisse Resultate, beispielsweise zur organisatorischen Unterstützung, sind spezifisch im Kontext dieses Spitals zu betrachten. Daher wäre für eine nachfolgende Studie der Einbezug mehrerer ähnlich großer Institutionen sinnvoll - vor allem, um die gesamtschweizerische Situation abzubilden.

\section{Implikationen für die Praxis}

Es gilt anzuerkennen, dass die Eltern eine große Verantwortung für das Wohl und die Sicherheit des hospitalisierten mehrfachbehinderten Kindes tragen, und in der Folge Hilfsangebote für das Wohlbefinden dieser Eltern an Bedeutung gewinnen. PFP können wesentlich dazu beitragen, dass Eltern ein Gefühl von Sicherheit entwickeln, dadurch Verantwortung abgeben und sich nicht zunehmend erschöpft fühlen. Dies gelingt aber nur, wenn eine Konti- 
nuität durch die PFP und außerordentliche Kompetenz gewährleistet sind, wie es beispielsweise das Organisationsmodell der Bezugspflege ermöglicht oder wie es Pflegefachpersonen mit einem Advanced-Practice-Hintergrund bieten. Eine Advanced-Practice-Nurse zeichnet sich durch fachliche Spezialisierung und erweiterte klinische Fertigkeiten aus. Sie arbeitet unterstützend für Patientengruppen mit chronisch-komplexem Krankheitsbild und optimierend für alle Beteiligten (Giger \& DeGeest, 2008). Eine Voraussetzung, die von den Eltern genannt wurde, jedoch für diese Patientengruppe im deutschsprachigen Raum noch geschaffen werden müsste.

Das Wissen über die individuelle Situation und die entsprechenden elterlichen Kompetenzen ist unabdingbar, da nicht jede Familie gleichermaßen Unterstützung benötigt. Erst eine vorausgehende Anamnese, wie sie Oulton, Sell, Kerry und Gibson (2014) empfehlen, ermöglicht eine individuelle Begleitung und Beratung sowie empathische Kommunikation und Information. Auch muss ein elterliches Mitspracherecht eingeräumt werden, sei es in Form von gemeinsamen Visiten, Pflegegesprächen und einem institutionellen Rahmen, in dem die Rolle, die Kompetenzen und die Form der elterlichen Mitsprache geklärt sind.

Zusammenfassend ist zu betonen, dass das Wohlergehen und die Kompetenzen der Eltern von mehrfachbehinderten Kindern im Spital zentral sind, da sie wiederum für das Wohl des Kindes von eminenter Bedeutung sind. Der Klärung der Frage, inwiefern PFP mit erweiterten Kompetenzen Eltern entlasten und befähigen, ist in weiteren Studien im pädiatrischen Kontext nachzugehen. Auch sind weiterführende Diskussionen und Studien über Modelle der Zusammenarbeit und Entlohnung der pflegenden Eltern im Spital erforderlich. Die vorliegende Studie hat einige Themenbereiche diskutiert, an die - so hoffen wir - mit weiteren Praxisentwicklungs- und Forschungsprojekten angeknüpft werden kann.

\section{Förderung}

Die vorliegende Arbeit wurde unterstützt von der Stiftung Anna Müller Grocholski und der Gruppe Reachout des Universitäts-Kinderspitals Zürich.

\section{Literatur}

Ausserhofer, D.; Mantovan, F.; Pirhofer, R.; Huber, M.; Them, C. (2009). The burden of parents caring for their children and adolescents with severe disabilities in South Tyrol. Pflege, 22 (3), $184-192$.

Avis, M.; Reardon, R. (2008). Understanding the views of parents of children with special needs about the nursing care their child receives when in hospital: a qualitative study. Journal of Child Health Care, 12 (1), 7 - 17.

Bell, J. M. (2014). Family centered care and family nursing: three beliefs that matter most. Pflege, 27 (4), 213 - 217.

Benner, P.; Wrubel, J. (1997). Pflege, Stress und Bewältigung. Bern: Hans Huber.
Büker, C. (2010). Leben mit einem behinderten Kind (1. Aufl.). Bern: Hans Huber.

Bundesamt für Statistik (2014). Medizinische Statistik der Krankenhäuser. @ BFS - Statistisches Lexikon der Schweiz, Neuchâtel.

Burns, K. H.; Casey, P. H.; Lyle, R. E.; Bird, T. M.; Fussell,J.J.; Robbins, J. M. (2010). Increasing prevalence of medically complex children in US hospitals. Pediatrics, 126 (4), 638-646.

Creswell, J. W.; Plano Clark, V. L. (2011). Design and conducting mixed methods research (2nd Edition). London: SAGE Publications, Inc.

Cunningham, B. J.; Rosenbaum, P. L. (2014). Measure of processes of care: a review of 20 years of research. Developmental Medicine and Child Neurology, 56 (5), $445-452$.

De Geeter, K. I.; Poppes, P.; Vlaskamp, C. (2002). Parents as experts: the position of parents of children with profound multiple disabilities. Child: Care, Health and Development, 28 (6), 443 - 453.

Flick, U.; von Kardoff, E.; Steinke, I. (2005). Qualitative Forschung. Ein Handbuch. (11. Aufl.). Berlin: Rowohlt.

Ford, K.; Turner, D. (2001). Stories seldom told: paediatric nurses' experiences of caring for hospitalized children with special needs and their families. Journal of Advanced Nursing, 33 (3), $288-295$.

Giger, M.; DeGeest, S. (2008). Neue Versorgungsmodelle und Kompetenzen sind gefragt. Schweizerische Ärztezeitung, 98 (43), $1839-1843$

Graham, R. J.; Pemstein, D. M.; Curley, M. A. (2009). Experiencing the pediatric intensive care unit: perspective from parents of children with severe antecedent disabilities. Critical Care Medicine, 37 (6), $2064-2070$.

Hemsley, B.; Kuek, M.; Bastock, K.; Scarinci, N.; Davidson, B. (2013). Parents and children with cerebral palsy discuss communication needs in hospital. Developmental Neurorehabilitation, 16 (6), $363-374$.

Houtrow, A. J.; Larson, K.; Olson, L. M.; Newacheck, P. W.; Halfon, N. (2014). Changing trends of childhood disability, 2001-2011. Pediatrics, 134 (3), $530-538$.

Iversen, A. S.; Graue, M.; Clare, J. (2009). Parents' perspectives of surgery for a child who has cerebral palsy. Journal of Pediatric Health Care, 23 (3), $165-172$.

Iversen, A. S.; Graue, M.; Raheim, M. (2013). At the edge of vulnerability - lived experience of parents of children with cerebral palsy going through surgery. International Journal of Qualitative Studies on Health and Well-being, 8,1-10.

Mayring, P. (2007). Qualitative Inhaltsanalyse. Grundlagen und Technik (Vol. 9). Basel: Beltz.

Mikkelsen, G.; Frederiksen, K. (2011). Family-centred care of children in hospital - a concept analysis. Journal of Advanced Nursing, 67 (5), $1152-1162$.

Olli, J.; Vehkakoski, T.; Salantera, S. (2014). The habilitation nursing of children with developmental disabilities - beyond traditional nursing practices and principles? International Journal of Qualitative Studies on Health and Well-being, 9, 23106.

Oulton, K.; Heyman, B. (2009). Devoted protection: How parents of children with severe learning disabilities manage risks. Health, Risk \& Society, 11 (4), $303-319$.

Oulton, K.; Sell, D.; Kerry, S.; Gibson, F. (2014). Individualizing hospital care for children and young people with learning disabilities: it's the little things that make the difference. Journal of Pediatric Nursing, 30 (1), $78-86$.

Phua, V.; Reid, S. M.; Walstab, J. E.; Reddihough, D. S. (2005). Inpatient care of children with cerebral palsy as perceived by their parents. Journal of Paediatrics and Child Health, 41 (8), $432-436$.

Ravens-Sieberer, U.; Morfeld, M.; Stein, R. E.; Jessop, D. J.; Bullinger, M.; Thyen, U. (2001). The testing and validation of the German version of the impact on family scale in families with children with disabilities. Psychotherapie Psychosomatik medizinische Psychologie, 51 (9-10), $384-393$.

Robinson, A.; Oxnam, C.; Kelly, S.; Broadbent, J.; Dillon, M. (1993). A study into nursing children with disabilities in an acute care context: The 3W Disability Awareness Group research report. Melbourne:The Royal Children's Hospital. 
Seliner, B.; Wattinger, A.; Spirig, R. (2015). Experiences and needs of parents of hospitalized children with disabilities and the health professionals responsible for the child's health-care: A systematic review. Pflege 28 (5), $263-276$.

Tadema, A. C.; Vlaskamp, C. (2010). The time and effort in taking care for children with profound intellectual and multiple disabilities: a study on care load and support. British Journal of Learning Disabilities, 38 (1), $41-48$.

Thyen, U.; Sperner, J.; Morfeld, M.; Meyer, C.; Ravens-Sieberer, U. (2003). Unmet health care needs and impact on families with children with disabilities in Germany. Ambulatory Pediatrics, 3 (2), $74-81$.

UDSmr. (2009). WeeFIM IITM System Klinisches Trainingshandbuch. WeeFIM $\|^{\circledR}$ CLINICAL GUIDE VERSION 6.0. (Translated Version 1.0. by N. Habenicht und B. Rückriem). Zürich: Universitäts-Kinderklinken Zürich, Rehabilitation.

Ware, J. (2000). SF-36 Health Survey Update. Spine, 25 (24), $3130-3139$.

WHO. (2010). International Classification of Functioning, Disability and Health (ICF). www.who.int/classifications/icf/en [11.3.2014].

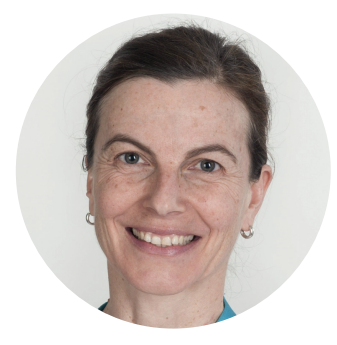

Brigitte Seliner, MNS, RN

Universitäts-Kinderspital

Zürich - Eleonorenstiftung

Rehabilitationszentrum für Kinder

und Jugendliche

Mühlebergstrasse 104

8919 Affoltern am Albis

Schweiz

brigitte.seliner@kispi.uzh.ch

\section{Was war die größte Herausforderung bei Ihrer Studie?}

Die Datenvielfalt zu reduzieren und dem Erleben und den Bedürfnissen den Eltern dennoch gerecht zu werden.

Was wünschen Sie sich bezüglich der Thematik für die Zukunft?

Unbedingt eine APN für Familien mit hospitalisierten Kindern mit Mehrfachbehinderungen.

Was empfehlen Sie den LeserInnen zum Weiterlesen/Vertiefen? Das erste Gedicht aus The Moth Eaten World von Suzanne Edison (Georgetown: Finishing Line Press, 2014).

Manuskripteingang: 05.08.2014

Manuskript angenommen: 22.12.2014 